\title{
Liquid 1-propanol studied by neutron scattering, near-infrared, and dielectric spectroscopy
}

\author{
P. Sillrén, ${ }^{1}$ A. Matic, ${ }^{1}$ M. Karlsson, ${ }^{1}$ M. Koza, ${ }^{2}$ M. Maccarini, ${ }^{2}$ P. Fouquet, ${ }^{2}$ M. Götz, ${ }^{3}$ \\ Th. Bauer, ${ }^{3}$ R. Gulich, ${ }^{3}$ P. Lunkenheimer, ${ }^{3}$ A. Loidl, ${ }^{3}$ J. Mattsson, ${ }^{4}$ C. Gainaru, ${ }^{5}$ \\ E. Vynokur, ${ }^{5}$ S. Schildmann, ${ }^{5}$ S. Bauer, ${ }^{5}$ and R. Böhmer ${ }^{5}$ \\ ${ }^{1}$ Department of Applied Physics, Chalmers University of Technology, SE-412 96 Göteborg, Sweden \\ ${ }^{2}$ Institut Laue Langevin, 6, rue Jules Horowitz, BP 156, 38042 Grenoble Cedex 9, France \\ ${ }^{3}$ Experimental Physics V, University of Augsburg, 86135 Augsburg, Germany \\ ${ }^{4}$ School of Physics and Astronomy, University of Leeds, Leeds LS2 9JT, United Kingdom \\ ${ }^{5}$ Fakultät für Physik, Technische Universität Dortmund, 44221 Dortmund, Germany
}

(Received 28 October 2013; accepted 4 March 2014; published online 24 March 2014)

\begin{abstract}
Liquid monohydroxy alcohols exhibit unusual dynamics related to their hydrogen bonding induced structures. The connection between structure and dynamics is studied for liquid 1-propanol using quasi-elastic neutron scattering, combining time-of-flight and neutron spin-echo techniques, with a focus on the dynamics at length scales corresponding to the main peak and the pre-peak of the structure factor. At the main peak, the structural relaxation times are probed. These correspond well to mechanical relaxation times calculated from literature data. At the pre-peak, corresponding to length scales related to H-bonded structures, the relaxation times are almost an order of magnitude longer. According to previous work [C. Gainaru, R. Meier, S. Schildmann, C. Lederle, W. Hiller, E. Rössler, and R. Böhmer, Phys. Rev. Lett. 105, 258303 (2010)] this time scale difference is connected to the average size of $\mathrm{H}$-bonded clusters. The relation between the relaxation times from neutron scattering and those determined from dielectric spectroscopy is discussed on the basis of broad-band permittivity data of 1-propanol. Moreover, in 1-propanol the dielectric relaxation strength as well as the near-infrared absorbance reveal anomalous behavior below ambient temperature. A corresponding feature could not be found in the polyalcohols propylene glycol and glycerol. () 2014 AIP Publishing LLC. [http://dx.doi.org/10.1063/1.4868556]
\end{abstract}

\section{INTRODUCTION}

The presence of hydroxyl groups (OH groups) in biological macromolecules, sugars, alcohols, as well as water has a strong impact on the function of the molecules and on the interactions with their surroundings. Monohydroxy alcohols, being among the simplest of OH-bonding molecules, provide us with the possibility to study the effect of hydrogen bonding on structure and dynamics by changing the length and the branching of the alkyl chain in the molecule. Each $\mathrm{OH}$ group in a monohydroxy alcohol can participate in three H-bonds, one involving the hydrogen, and two involving the oxygen. The molecular clusters formed through H-bonding are believed to involve either chain- or tree-like structures, ${ }^{1-15}$ or closed loops and rings. ${ }^{16-18}$ Recent nuclear magnetic resonance (NMR) and neutron diffraction studies on alcohols for which the $\mathrm{OH}$ group is located in a terminal site support a description of the structure in terms of chains with a slight branching. ${ }^{2,19}$ For alcohols for which the $\mathrm{OH}$ group is located in a non-terminal position ring-like structures are often found to be preferred. ${ }^{20-23}$

Monohydroxy alcohols, just like all other liquids, show a structural relaxation process also called $\alpha$-relaxation. The $\alpha$ process is resolved by a number of experimental techniques such as dielectric ${ }^{24-26}$ and mechanical spectroscopy ${ }^{27}$ as well as calorimetry ${ }^{28}$ and light scattering. ${ }^{29}$ For monohydroxy alcohols the $\alpha$-process has been shown to be related to the motion of the molecular alkyl chain., 2,30,31

In addition to the $\alpha$-process, the H-bonded clusters of monohydroxy alcohols are believed to cause a relaxation process that is slower than the structural relaxation and is particularly prominent in the dielectric response. ${ }^{24,29,32,33}$ This process, first analyzed in detail by Debye, ${ }^{34}$ and hence referred to as the Debye process, corresponds to a single exponential form of the underlying autocorrelation function. The Debye process has been a controversial subject, and although significant progress has been made, its origin and connection to more elementary relaxations is still not fully understood. ${ }^{2,35}$ One of the reasons for this situation may be that while this process is prominent in dielectric measurements, other techniques such as neutron scattering, ${ }^{36}$ Brillouin scattering, ${ }^{29,37,38}$ ultrasonic techniques, ${ }^{39}$ and photon correlation spectroscopy ${ }^{29}$ were reported to be insensitive to it. Shear mechanical experiments demonstrate, however, that the Debye process is indeed accompanied by a mechanical signature. ${ }^{40,41}$

Finally, NMR spectroscopy reveals a third relaxation process, intermediate between the $\alpha$ - and the Debye relaxation process which is related to the time a given $\mathrm{OH}$ group stays within a hydrogen bonded cluster. ${ }^{2,42}$

In this work, we characterize the relaxation processes in the monohydroxy alcohol 1-propanol using quasi-elastic 


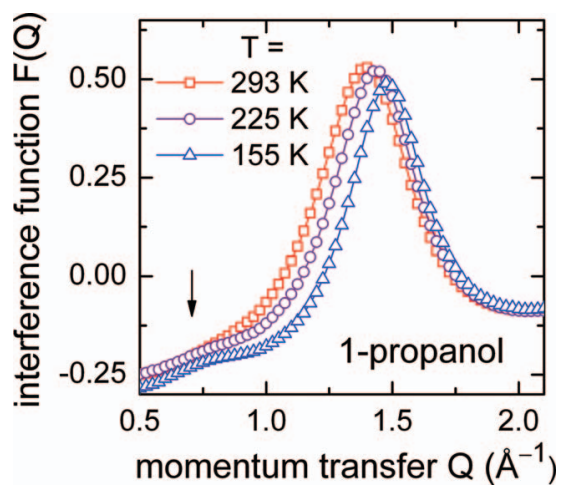

FIG. 1. Total interference functions, $F(Q)$, measured for propanol-d $\mathrm{d}_{7}$ using the NIMROD diffractometer at the ISIS spallation neutron source. The prepeak position is highlighted by an arrow. More details can be found in Ref. 19 from which this figure was adapted.

neutron scattering. By combining two instruments, the time-of-flight spectrometer IN6 for the faster dynamics, and the neutron spin-echo instrument IN11C for the slower part of the dynamics, we cover four orders of magnitude in time and simultaneously length scales ranging from those of single molecules to those corresponding to hydrogen bonded intermolecular networks. The latter length scale is believed to be at the origin of a pre-peak observed in the static structure factor, $S(Q){ }^{43,44}$ Corresponding total neutron interference functions, $F(Q)$, which essentially reflect $S(Q)$ were taken from Ref. 19 and are shown in Fig. 1. One recognizes that in addition to the main peak at momentum transfers $Q \approx 1.4 \AA^{-1}$, a prepeak appears at $Q \approx 0.7 \AA^{-1}$. Its intensity is rather small which, in the framework of empirical potential structure refinement calculations, was rationalized by referring to the small relative weight of the hydroxyl-related partial structure factors with respect to those characterizing the alkyl chain. ${ }^{19}$ That the prepeak is due to supramolecular structure formation induced by hydrogen bonding is backed up experimentally by comparative neutron structure factor measurements on 2-propanol versus 2-chloro- and 2-bromo-propane. ${ }^{45}$ In those studies a prepeak was detected for the monohydroxy alcohol but not for the halogenated analogues. The observation of prepeaks is, however, not unique to monohydroxy alcohols but was reported also for other hydrogen bonded liquids, see Ref. 46 for a recent example and Ref. 47 for further references.

To enable a detailed comparison of the neutron results with those from other techniques broad-band dielectric measurements on 1-propanol are performed with particular emphasis on the high-frequency regime, providing spectra in a wider frequency range than in previous investigations. ${ }^{29,48-51}$ Importantly, our dielectric experiments allow us to assess in detail the uncertainty in the determination of the structural relaxation times in the microwave region. We find that the dielectric relaxation strength and the near-infrared (NIR) absorbance of 1-propanol shows anomalous behavior below ambient temperature. To check whether such absorbance behavior is characteristic only of monohydroxy alcohols or of hydrogen bonded systems in general, we perform an NIR study of the corresponding polyalcohols propylene glycol $(1,2 \text {-propanediol })^{52}$ and glycerol (1,2,3-propanetriol). ${ }^{53}$

\section{EXPERIMENTAL DETAILS AND DATA TREATMENT}

\section{A. Quasielastic neutron scattering and neutron spin echo experiments}

The experiments were performed on the time-of-flight spectrometer IN6 and on the IN11C neutron spin-echo instrument at the Institut Laue Langevin (ILL), Grenoble, France. Data were recorded for six temperatures in the range from 161 to $314 \mathrm{~K}$. The time window of the combined IN6/IN11 data covers $0.2-2000 \mathrm{ps}$ and the momentum transfer range was from $Q=0.3$ to $2.5 \AA^{-1}$ on IN6 and 0.456 to 1.02 and 1.14 to $1.62 \AA^{-1}$ on IN11. The instrumental resolution functions were determined from measurements on a sample held at $1.5 \mathrm{~K}$ for which only static scattering is present.

The IN11 data were treated using standard scripts in the Intercept Ground Optical Recorder (IGOR) PRO software and the IN6 data using the lamp software, both provided by the ILL. The data were binned with regards to momentum transfer within two regions corresponding to the value of the pre-peak $\left(0.7 \AA^{-1}\right)$ and the main peak $\left(1.4 \AA^{-1}\right)$ of the static structure factor. The intermediate scattering functions, $S(Q, t)$, are directly obtained from the corrected IN11 data, whereas the IN6 data were Fourier transformed to obtain $S(Q, t)$. For both neutron experiments the samples were contained within a concentric aluminum cylinder, $60 \mathrm{~mm}$ high with an outer diameter of $22 \mathrm{~mm}$. The inner diameter of the cylinder was $20 \mathrm{~mm}$, calculated to make sure that about $10 \%$ of the incident neutrons were scattered by the sample, thus reducing multiple scattering to less than $1 \%$.

Deuterated 1-propanol (CDN isotopes, Canada, specified to be $98 \%$ isotopically enriched) was used as received. The deuteration degree was determined to $95 \%$ using infrared spectroscopy. The fraction of coherent scattering from a $100 \%$ deuterated 1-propanol would have been $80 \%$, in our case it was close to $76 \%$.

\section{B. Broad-band dielectric measurements}

For the dielectric measurements, three experimental techniques were combined to obtain broad-band dielectric spectra of the complex permittivity covering the frequency range from $0.1 \mathrm{~Hz}$ to $40 \mathrm{GHz}$. In the low-frequency range, $v<3 \mathrm{MHz}$, a frequency-response analyzer (Novocontrol Alpha-analyzer) was used. Measurements in the radiofrequency and microwave ranges $(1 \mathrm{MHz}<v<3 \mathrm{GHz})$ were performed using a reflectometric technique with the sample capacitor mounted at the end of a coaxial line. ${ }^{54}$ For these measurements an Agilent E4991A impedance analyzer was employed. For both methods, the sample material from Aldrich (stated purity: 99.7\%) was filled into parallel-plate capacitors with plate distances of 0.1 to $0.15 \mathrm{~mm}$. The results at the highest frequencies, $100 \mathrm{MHz}-40 \mathrm{GHz}$, were obtained by measuring the reflection coefficient of an open-ended coaxial line, directly immersed into the sample liquid. ${ }^{55,56}$ For this purpose, an Agilent "Dielectric Probe Kit" and an Agilent E8363B Network Analyzer were used. For cooling and heating, the samples were put into a nitrogen-gas cryostat (Novocontrol Quatro). 
Due to uncertainties in the geometrical and stray capacitances, it is usually necessary to shift the permittivity values obtained by different experimental techniques with respect to each other to obtain continuous broadband spectra. Here, the open-end coaxial technique used at the highest frequencies does not suffer from such problems and was used to fix the absolute values of the permittivity at lower frequencies. It is important to note that data sets obtained with one experimental set-up are shifted by a single scaling factor only, which depends neither on frequency nor on temperature. The reasonable match of the three data sets obtained in this way and the overlap of frequency ranges of the different devices helps to exclude any ambiguities that may arise from the employed data correction.

\section{Near-infrared spectroscopy}

Near-infrared spectra were recorded using a Varian CARY 2300 photospectrometer as described in Ref. 23. In addition to 1-propanol (from Sigma-Aldrich, stated purity: 99.7\%), we also studied propylene glycol (from Fluka, stated purity: 99.5\%) and glycerol (from Fluka, stated purity: 99.5\%) using NIR measurements. All samples were used as received.

\section{RESULTS}

\section{A. Neutron scattering}

Normalized intermediate scattering functions $S(Q, t) / S(Q, 0)$ for $Q$ values around the pre-peak $\left(0.7 \AA^{-1}\right)$ and around the main peak $\left(1.4 \AA^{-1}\right)$ of the static structure factor are shown in Fig. 2. The IN6 data cover the shorter time scales $\left(2 \times 10^{-13} \mathrm{~s}<t<10^{-11} \mathrm{~s}\right)$ and the IN11 data the longer time scales $\left(5 \times 10^{-12} \mathrm{~s}<t<2 \times 10^{-9} \mathrm{~s}\right)$. To obtain a good match between the two data sets the IN11 data were scaled by factors ranging from 1.35 to 1.8. Directly from the experimental data we observe a slowing down of the dynamics with decreasing temperature. It is also clear that the dynamics is significantly slower at low momentum transfers, $Q=0.7 \AA^{-1}$, as compared to those at $Q=1.4 \AA^{-1} .{ }^{57}$

To analyze the data quantitatively, they were fit using a sum of an exponential function and a stretched exponential function according to

$$
S(Q, t) / S(Q, 0)=A \mathrm{e}^{-t / \tau_{1}}+(1-A) \mathrm{e}^{-\left(t / \tau_{2}\right)^{\beta_{2}}} .
$$

This approximation should be valid when the time scales $\tau_{1}$ and $\tau_{2}$ are sufficiently well separated. In Fig. 2 the lines represent best fits to the data. Fits were not performed for the two lowest temperatures for the low $Q$ setting, where reliable results could not be obtained.

The first single exponential function in Eq. (1) describes the initial fast decay of the relaxation function on a time scale, $\tau_{1}$, around $1 \mathrm{ps}$. This process shows only a weak temperature dependence and its origin is assigned to microscopic motions. The time scale $\tau_{2}$ turned out to be at least 10 times longer than $\tau_{1}$ and therefore, in the following, we focus our analysis on the slower process. The parameters $\tau_{2}$ and $\beta_{2}$ characterizing the stretched exponential relaxation function in Eq. (1) were

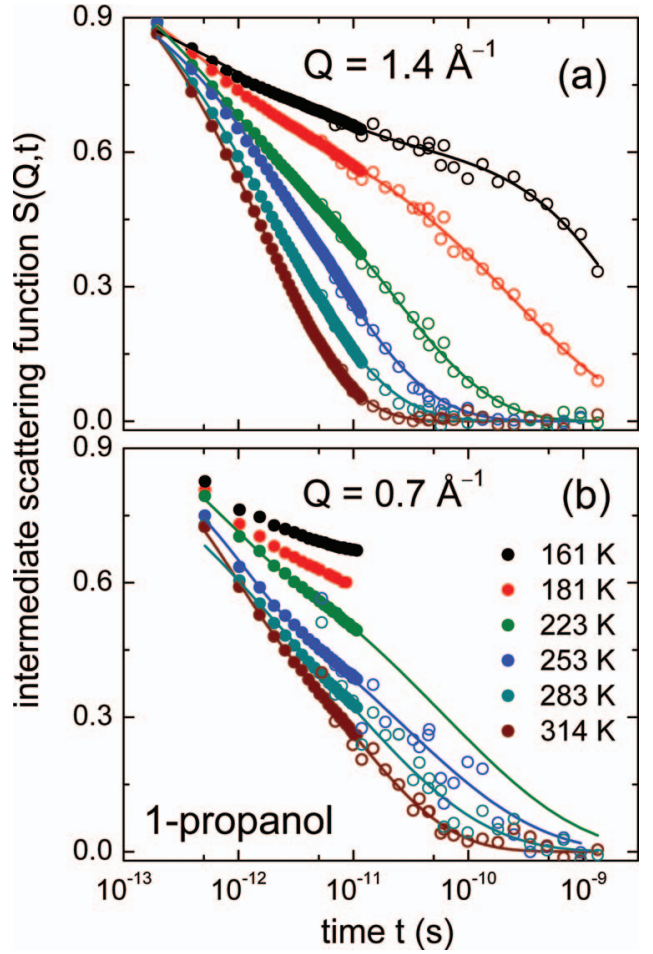

FIG. 2. Normalized intermediate scattering functions $S(Q, t) / S(Q, 0)$ from IN6 (solid symbols, short times) and IN11 (open symbols, long times) for different temperatures are represented as symbols. Frame (a) shows relaxations around the main peak of the static structure factor, while frame (b) shows relaxations around the pre-peak. Best fits using Eq. (1) are given as lines.

determined for several temperatures and momentum transfers. We found that the stretching parameter $\beta_{2}$ increases with increasing temperature. It evolves from around 0.3 at low temperatures and reaches 0.7 at high temperatures for $Q=1.4$ $\AA^{-1}$; for $Q=0.7 \AA^{-1}$ the exponent $\beta_{2}$ increases from 0.3 to 0.5 in the accessible $T$ range. Overall, as a function of $Q$ the stretching exponents displayed large, but not recognizably systematic variations (up to \pm 0.1 ). As a function of temperature the $\beta_{2}$ exponents at fixed $Q$ are much smaller, except for $223 \mathrm{~K}$ and at low $Q$. Here, IN11 data are not available and fits were thus performed using IN6 data, only. The value for $\tau_{2}$ we obtain under these conditions is estimated to be reliable within a factor of about 4 .

The explicit temperature dependence of $\tau_{2}$ as measured at the main-peak, discussed in detail in Sec. IV A, is typical for a structural relaxation process. Let us emphasize that the slowing down of the relaxation around the pre-peak, also dealt with more thoroughly in Sec. IV A, hints at the existence of structures at length scales $2 \pi / 0.7 \AA^{-1} \approx 9 \AA$ (corresponding to supramolecular structures comprising 3 to 5 molecules) that influence the dynamics. It was noted already in 1927 that the distance $2 \pi / Q$, corresponding to the pre-peak, increases linearly with increasing alkyl chain length ${ }^{43}$ suggesting that the pre-peak is related to the mean distance between adjacent clusters. Our data thus suggest that the inter cluster correlation survives longer than the correlations between single molecules, in agreement with the transient chain model. ${ }^{2}$ Within that approach the movement of the supramolecular structure is caused mainly by monomers joining and 
leaving the cluster, rather than by movement of the clusters as a whole.

\section{B. Dielectric spectroscopy}

Due to the large dipole moment of the monohydroxy alcohol molecules, their dynamics are profitably studied using dielectric spectroscopy. We have carried out such measurements with particular emphasis on the high-frequency range to enable detailed comparisons with the results from neutron scattering and also to explore possible limitations of such comparisons.

Fig. 3 shows the frequency dependent dielectric constant $\varepsilon^{\prime}$ (a) and dielectric loss $\varepsilon^{\prime \prime}$ (b) of 1-propanol for selected temperatures. The spectra are dominated by the typical signatures of a relaxation process: a step-like decrease of $\varepsilon^{\prime}(v)$ with increasing frequency and a peak in $\varepsilon^{\prime \prime}(v)$, which both shift through the experimental frequency window as a function of temperature. By comparing the corresponding relaxation times with those obtained by other experimental methods, Hansen et al. ${ }^{29}$ demonstrated that the most prominent relaxation process of 1-propanol does not correspond to the structural $\alpha$ relaxation. Instead, it corresponds to the Debye process which is generally found in monohydroxy alcohols.

A closer look at Fig. 3 reveals the presence of two additional relaxation processes. In Fig. 3(a), each curve seems to be composed of two relaxation steps, the second step corresponding to a decay of $\varepsilon^{\prime}(v)$ from about 10 to 4 . The corresponding peak in $\varepsilon^{\prime \prime}$ is superimposed by the main relaxation peak and shows up as shoulder at the high-frequency flank of
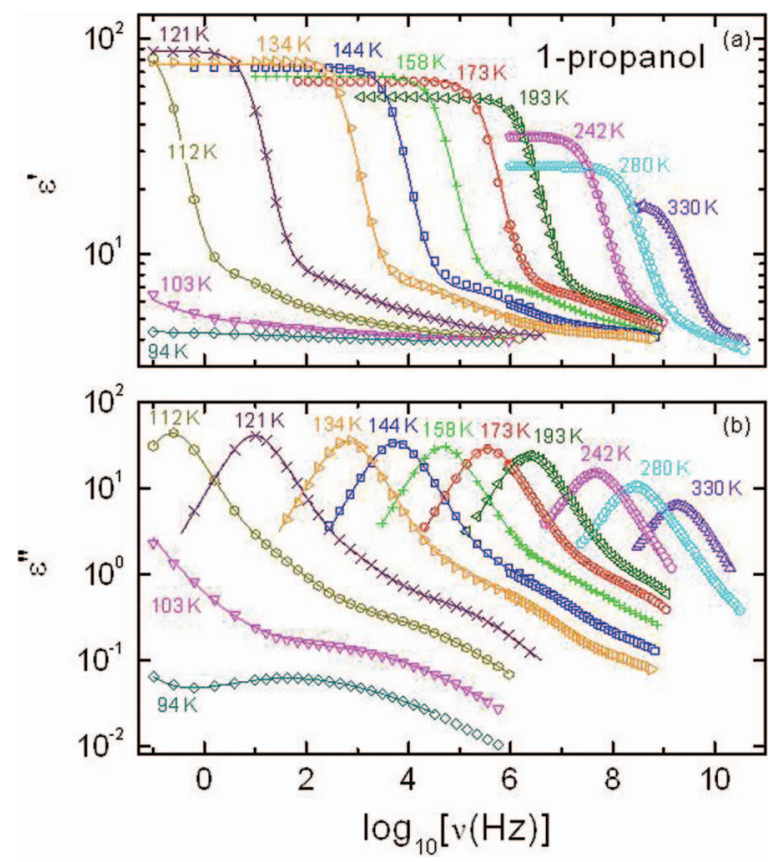

FIG. 3. Spectra of the dielectric constant (a) and loss (b) of 1-propanol shown for selected temperatures. The data were measured using three experimental setups: frequency-response analysis (typical frequency range $0.1 \mathrm{~Hz}<v<2.4 \mathrm{MHz}$ ), coaxial reflection technique $(1 \mathrm{MHz}<v<1.5$ $\mathrm{GHz}$ ), and open-ended coaxial-line technique $(300 \mathrm{MHz}<v<30 \mathrm{GHz}$ ); see Sec. II B for details. The lines are fits using Eq. (2), simultaneously performed for the real and the imaginary part. the main peak, e.g., at around $100 \mathrm{~Hz}$ for $112 \mathrm{~K}$ in Fig. 3(b). This process has been identified with the $\alpha$ relaxation (see Ref. 29). Moreover, at the lowest temperatures a relatively small peak reveals the presence of a third relaxation process, which successively merges with the other relaxation features with increasing temperature. The corresponding step in $\varepsilon^{\prime}(v)$ is too small to become visible within the scale of Fig. 3(a). This peak arises from a secondary relaxation, usually termed Johari-Goldstein $\beta$ relaxation, which is often found in glassforming liquids. ${ }^{58-61}$ The dielectric response of 1-propanol as shown in Fig. 3 is consistent with the previously reported behavior ${ }^{29,48,49}$ but the present spectra cover a significantly broader frequency range.

As revealed by Fig. 3, the amplitudes of the steps in $\varepsilon^{\prime}$ and of the peaks in $\varepsilon^{\prime \prime}$ decrease with increasing temperature. A gradual decrease of the relaxation strength is found in most glass forming liquids. ${ }^{62}$ In principle, this can be understood in terms of the time-honored Onsager theory ${ }^{63}$ However, already a simple inspection of Fig. 3 reveals that above about $240 \mathrm{~K}$ this decrease becomes significantly stronger (see Sec. IV C for further discussion). Such an unusual temperature development of the relaxation strength at high temperatures was recently demonstrated for a variety of monohydroxy alcohols. ${ }^{23,64}$

The lines in Fig. 3 are fits using a sum of three relaxation functions to account for the Debye, $\alpha$, and $\beta$ relaxation, simultaneously performed for the real and the imaginary part of the permittivity. For the $\alpha$ and $\beta$ relaxations, the empirical Cole-Davidson (CD) or Cole-Cole (CC) functions were used, respectively, ${ }^{65}$ overall leading to

$$
\begin{aligned}
\varepsilon^{*}(\nu)= & \varepsilon_{\infty}+\frac{\Delta \varepsilon_{D}}{1+2 \pi i \nu \tau_{D}}+\frac{\Delta \varepsilon_{\alpha}}{\left[1+\left(2 \pi i \nu \tau_{\alpha}\right)\right]^{\beta_{\alpha}}} \\
& +\frac{\Delta \varepsilon_{\beta}}{1+\left(2 \pi i \nu \tau_{\beta}\right)^{\alpha_{\beta}}} .
\end{aligned}
$$

Here, $\varepsilon_{\infty}$ is the high-frequency dielectric constant, $\Delta \varepsilon_{D, \alpha, \beta}$ denote the relaxation strengths of the corresponding processes, $\tau_{D, \alpha, \beta}$ are the relaxation times, and $\beta_{\alpha}$ and $\alpha_{\beta}$ are the width parameters of the $\mathrm{CD}$ and $\mathrm{CC}$ function, respectively. These fits lead to a reasonable description of the measured broadband spectra. For temperatures $T \geq 134 \mathrm{~K}$, for which the $\beta$ relaxation is not clearly visible, the last term in Eq. (2) was omitted. However, one should be aware that especially at the higher temperatures, the parameters obtained for the $\alpha$ relaxation are of limited significance because some of the corresponding spectral features are strongly superimposed by the Debye process. ${ }^{66}$

Fig. 4 shows the experimental spectra at two selected temperatures together with several alternative fit curves. While the black lines represent the best fits as also shown in Fig. 3, the colored curves are fits with $\tau_{\alpha}$ fixed to lower values than obtained from the free fits. For the red curves in frames (a) and (b), marked deviations of fit and experimental data show up. In contrast, the deviations of the green curves are still tolerable. The comparison of fit curves as shown in Fig. 4 was used to estimate the error bars for $\tau_{\alpha}$. Overall, this figure demonstrates that $\tau_{\alpha}$ deduced from the fits can have a rather high uncertainty, especially at the higher 


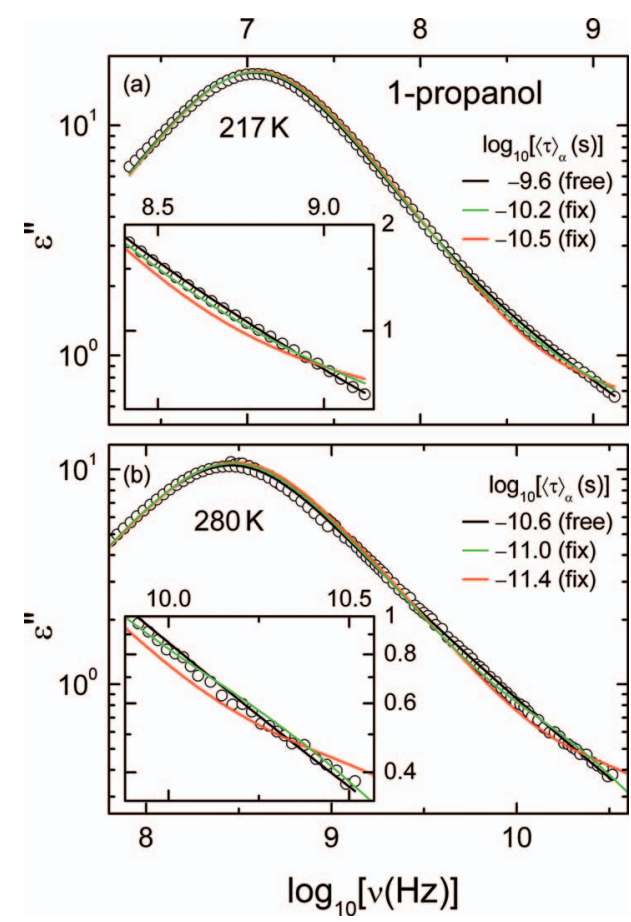

FIG. 4. Dielectric loss spectra of 1-propanol at two selected temperatures (the insets show a zoomed view of the high-frequency region). The black lines are the best fits of the experimental data using Eq. (2) (not including the term referring to the $\beta$ relaxation) as already shown in Fig. 3(b). The colored lines are fits with $\tau_{\alpha}$ fixed to lower values than obtained from the free fits, providing an estimate for the significance of $\tau_{\alpha}$ deduced from the fits. The fixed value of $\tau_{\alpha}$ used for the green line in (a), still leading to a reasonable fit, corresponds to the lower limit of the error bar at $217 \mathrm{~K}\left(1000 / T=4.61 \mathrm{~K}^{-1}\right.$, cf. Fig. 8).

temperatures, for which the $\alpha$ peak has almost merged with the much stronger Debye peak (cf. Fig. 3 and the discussion in Sec. IV A). In contrast, at lower temperatures $\tau_{\alpha}$ can be determined with higher precision.

\section{Near-infrared spectroscopy}

Previously, the anomalous behavior of the dielectric relaxation strength of the main relaxation process was found to be paralleled by the temperature dependence of the NIR absorbance arising from the $\mathrm{OH}$ bands for several long-chain monohydroxy alcohols. ${ }^{23}$ In order to find out whether similar observations can be made also for 1-propanol and whether it is shared by polyalcohols as well, we studied alcohols with 1 , 2 , and 3 hydroxyl groups.

In Fig. 5 we present NIR spectra for wavelengths from 1320 to $1700 \mathrm{~nm}$ for which the first overtone of the $\mathrm{OH}$ stretching vibrations of 1-propanol, propylene glycol, and glycerol dominates. In Fig. 5(a) spectra recorded at a temperature of $300 \mathrm{~K}$ are summarized for these three substances. One recognizes that the broad array of bands showing up in the 1400 to $1600 \mathrm{~nm}$ range roughly scales with the density of the absorbing species, the $\mathrm{OH}$ groups, as expected. A glance at the temperature dependent absorbances of these substances, presented in Figs. 5(b)-5(d), reveals, however, significant differences in their thermal evolution. The spectra of 1-propanol display the typical NIR features ${ }^{67}$ observed

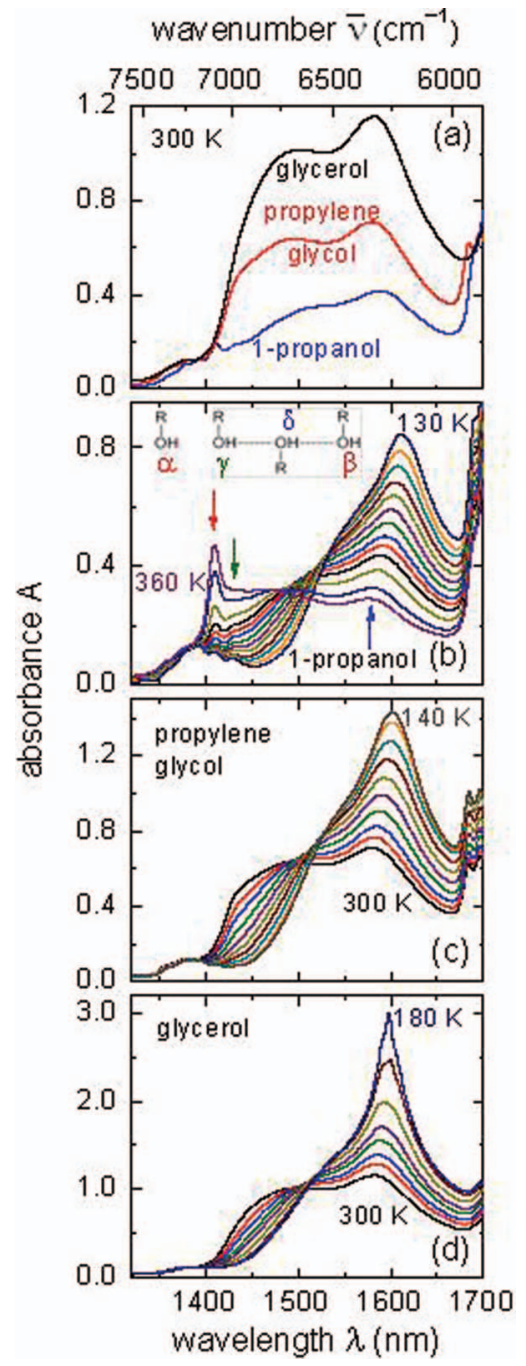

FIG. 5. NIR spectra of 1-propanol, propylene glycol, and glycerol. (a) Comparison of the absorbances of these substances at $300 \mathrm{~K}$. (b) Spectra of 1propanol taken at 360, 345, 320, 300, 285, 270, 250, 230, 210, 190, 170, 150, and $130 \mathrm{~K}$. The inset to panel (b) illustrates the assignment and nomenclature of the monomer and polymer bands usually applied to monohydroxy alcohols. The arrows mark the spectral position of the various bands. (c) Spectra of propylene glycol recorded at the same temperatures as for 1-propanol except for the lowest one, $140 \mathrm{~K}$, and (d) spectra of glycerol taken at 300, 280, $265,245,230,210,190$, and $180 \mathrm{~K}$.

for various other monohydroxy alcohols. ${ }^{23}$ This includes a narrow, so-called $\alpha / \beta$-band near $1410 \mathrm{~nm}$ which stems from weakly bonded $(\beta)$, proton accepting or non-bonded $(\alpha) \mathrm{OH}$ groups. Its temperature dependent intensity is superimposed onto a $T$ independent $\mathrm{CH}$ combination band. ${ }^{68}$ The broad spectral absorbance peak centered near $1600 \mathrm{~nm}$ marks the socalled polymer- or $\delta$-band originating from hydroxyl groups of strongly hydrogen bonded 1-propanol molecules which are not in a terminal position of a cluster. The assignment of these bands, including the very weak $\gamma$-band near $1430 \mathrm{~nm}$, is illustrated in the inset of Fig. 5(b). ${ }^{69}$

Let us now turn to the thermal evolution of the polyalcohol spectra. Near $1575 \mathrm{~nm}$ an absorbance peak is observed for propylene glycol and glycerol which strongly grows in intensity with decreasing temperatures. It is ascribed to intermolecularly bonded $\mathrm{OH}$ groups, ${ }^{53}$ and can be viewed to be 


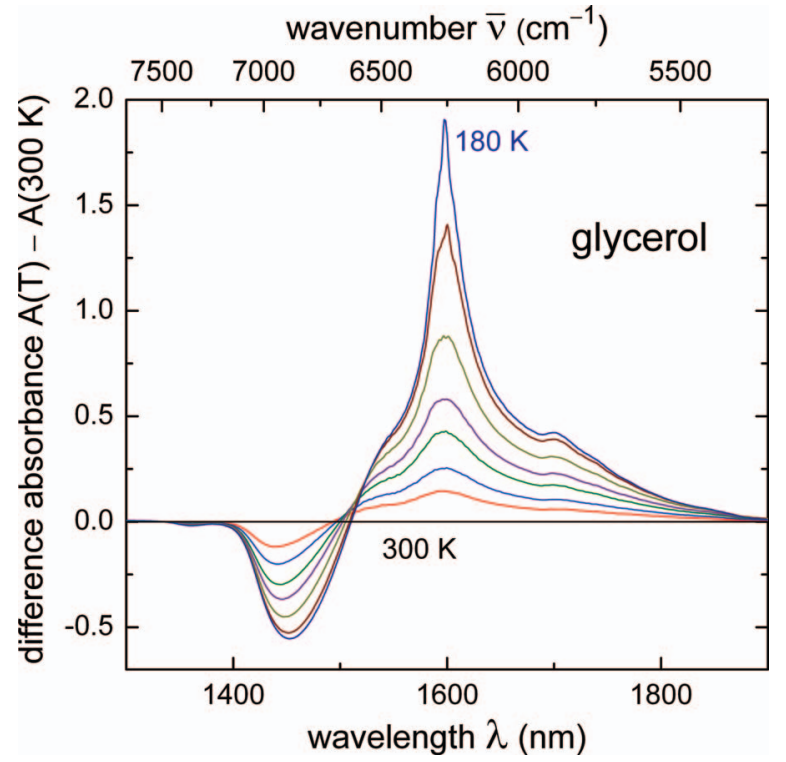

FIG. 6. Temperature dependent difference absorbance spectra, $A(\lambda, T)-$ $A(\lambda, 300 \mathrm{~K})$, of glycerol in the temperature range from 300 to $180 \mathrm{~K}$ at the same temperatures as in Fig. 5(d). By definition the difference spectrum at $300 \mathrm{~K}$ is zero.

analogous to the $\delta$-band in 1-propanol. A peaked $\alpha / \beta$ band is, however, not distinguishable in the spectra of the polyhydric alcohols. Merely, absorption is observed for $\lambda$ in the range from about 1400 to $1500 \mathrm{~nm}$ which appears to shift strongly to larger wavelengths as temperature decreases. A somewhat different impression arises if one considers difference spectra, $A(\lambda, T)-A\left(\lambda, T_{\text {ref }}\right.$ ). Using an (arbitrary) reference temperature, $T_{\text {ref }}=300 \mathrm{~K}$, this is exemplified for glycerol in Fig. 6 where two bands can clearly be identified: a gain peak of intermolecular origin near $1600 \mathrm{~nm}$ and an absorbance loss peak near 1440 to $1455 \mathrm{~nm}$ which might by assigned to nonbonded $\mathrm{OH}$ groups. ${ }^{53}$ The difference spectra (looking qualitatively similar to those of propylene glycol, not shown) clearly reveal opposing temperature dependences of the two bands. While for a quantitative analysis the difference spectra appear most straightforward, we note that the lower-wavelength feature can be analyzed also from the $A(\lambda, T)$ spectra directly. We found that, e.g., by integrating the spectral intensity between 1390 and $1530 \mathrm{~nm}$, cf. Fig. 5(c), the same trends are obtained as by analyzing the difference intensity between 1440 and 1455 nm, see Fig. 6.

In Fig. 7 we show the temperature dependent absorbance ratio, $A\left(\lambda_{\alpha / \beta}\right) / A\left(\lambda_{\delta}\right)$ for 1-propanol and $A\left(\lambda_{\mathrm{nb}}\right) / A\left(\lambda_{\delta}\right)$ for the polyalcohols. Here $A\left(\lambda_{\mathrm{nb}}\right)$ denotes the absorbance of nonor weakly bonded OH groups. In the form of a van't Hoff plot, Fig. 7 reveals a striking difference between this ratio for 1-propanol on the one hand and for the polyalcohols on the other. For 1-propanol the temperature dependence of $A\left(\lambda_{\alpha / \beta}\right) / A\left(\lambda_{\delta}\right)$ displays a major change, cf. Fig. 7(a). This was similarly observed for several other monohydroxy alcohols with different alkyl chain lengths. ${ }^{23}$ This anomalous behavior of 1-propanol is neither related to its melting point, indicated by the arrow in Fig. 7(a), nor to its much lower calorimetric glass transition temperature $\left(T_{g}^{\text {cal }} \approx 96 \mathrm{~K}\right){ }^{70}$

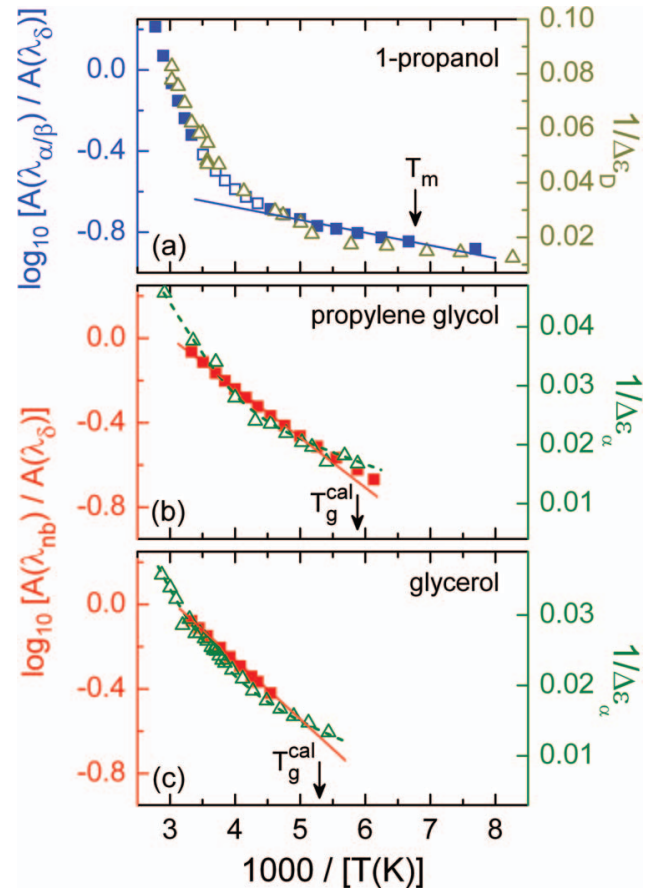

FIG. 7. Van't Hoff plot for the absorbance ratio from the present work represented as squares (scale on the left-hand side) of (a) 1-propanol, (b) propylene glycol, and (c) glycerol. The scale on the right-hand side refers to the dielectric relaxation strength $\Delta \varepsilon$ represented as triangles (1-propanol: present work, propylene glycol: Ref. 72, glycerol: Ref. 75), scaled to enable a comparison with the absorbance ratio. Solid lines represent fits with Eq. (3) using the enthalpies $\Delta H$ given in Table I. Dashed lines are guides for the eyes. The data represented by open squares in frame (a) were not used for the fitting. The arrows indicate the calorimetric glass transition temperatures, $T_{g}^{\mathrm{cal}}$, of propylene glycol and glycerol as well as the melting point, $T_{m}$, of 1-propanol. Obviously, the change in the absorbance or the dielectric relaxation strength is completely unrelated to $T_{m}$.

As mentioned above, the relaxation strength $\Delta \varepsilon_{D}$ of the Debye process determined from dielectric spectroscopy shows an unusually strong variation at high temperatures (Fig. 3). In Fig. 7(a), $1 / \Delta \varepsilon_{D}$ (right ordinate) as deduced from fits of the dielectric spectra with Eq. (2) is compared to the absorbance ratio from the infrared experiments (left ordinate). Similar to the findings for various other monohydroxy alcohols, ${ }^{23,64} \Delta \varepsilon_{D}$ and $A\left(\lambda_{\alpha / \beta}\right) / A\left(\lambda_{\delta}\right)$ can well be matched by choosing a proper scaling of the ordinates. Thus, $\Delta \varepsilon_{D}$ is directly correlated with the ratio of broken versus intact $\mathrm{H}$ bonds and its strong reduction at high temperatures can be ascribed to the breaking of hydrogen bonds.

For the polyhydric alcohols, a change in the temperature dependent absorbance ratio, as observed for 1propanol, is not discernible. Also sorbitol, a polyalcohol which contains six $\mathrm{OH}$ groups, lacks such a feature. ${ }^{71}$ For propylene glycol a slightly curved behavior is observed in the $\log _{10}\left[A\left(\lambda_{\mathrm{nb}}\right) / A\left(\lambda_{\delta}\right)\right]$ vs. $1 / T$ plot, see Fig. $7(\mathrm{c})$, but a clear change of behavior appears only near its $T_{g}^{\text {cal }}$ $\approx 170 \mathrm{~K},{ }^{72}$ similar to observations made for other glass formers. ${ }^{64,73}$ Glycerol, for which NIR data were recorded mainly above its $T_{g}^{\text {cal }}$ of about $189 \mathrm{~K},{ }^{74}$ yields a straight line in the representation of Fig. 7.

In Figs. 7(b) and 7(c), we show the inverse relaxation strengths of the main relaxation process (the $\alpha$-relaxation) 
TABLE I. Enthalpy changes $\Delta H$ determined from NIR spectroscopy for various alcohols containing $n$ hydroxyl groups per molecule. Data for sorbitol are from Ref. 71 and the other results are from the present work. The temperature range in which the enthalpy changes are obtained is indicated.

\begin{tabular}{lcccc}
\hline \hline Substance & $n$ & $T(\mathrm{~K})$ & $\Delta H(\mathrm{~kJ} / \mathrm{mol})$ & $\Delta H / n(\mathrm{~kJ} / \mathrm{mol})$ \\
\hline 1-propanol & 1 & $300-360$ & $\sim 20$ & $\sim 20$ \\
& & $130-220$ & 1.2 & 1.2 \\
Propylene glycol & 2 & $140-300$ & 4.1 & 2.0 \\
Glycerol & 3 & $180-300$ & 5.7 & 1.9 \\
Sorbitol & 6 & $250-500$ & 10.9 & 1.8 \\
\hline \hline
\end{tabular}

for propylene glycol ${ }^{72}$ and glycerol ${ }^{75}$ to provide a comparison with the corresponding absorbance ratios. We find that, irrespective of the chosen ordinate scaling, a satisfactory match of the temperature dependences of both quantities is not possible as their temperature dependence differs qualitatively. Obviously, in contrast to the monohydroxy alcohols, in these polyalcohols the amplitude of the main relaxation process is not directly related to the number of intact hydrogen bonds. This finding further confirms the different microscopic origins of the main relaxation processes in these two classes of alcohols.

For a quantitative analysis of the data shown in Fig. 7, let us assume that a two-state equilibrium exists involving the strongly hydrogen bonded and the non-bonded species. Then, from $\log _{10}\left[A\left(\lambda_{\alpha / \beta}\right) / A\left(\lambda_{\delta}\right)\right]$ for 1-propanol or $\log _{10}\left[A\left(\lambda_{\mathrm{nb}}\right) / A\left(\lambda_{\delta}\right)\right]$ for the polyalcohols, the enthalpy change $\Delta H$ characterizing the species participating in the assumed two-state equilibrium can be determined from $^{76}$

$$
\log _{10} \frac{A\left(\lambda_{\alpha / \beta}, \lambda_{\mathrm{nb}}\right)}{A\left(\lambda_{\delta}\right)}=-\frac{\Delta H}{R \ln (10)} \cdot \frac{1}{T}+\text { const. }
$$

Here $R$ designates the ideal gas constant. The solid lines in Fig. 7 reflect fits using Eq. (3) and yield the enthalpies summarized in Table I. For comparison, data for another hydrogen bonded liquid, sorbitol, is included. ${ }^{71}$ The enthalpies characterizing the polyhydric alcohols depend on the number $n$ of molecular $\mathrm{OH}$ groups.

\section{DISCUSSION}

\section{A. Temperature dependent time scales}

In Fig. 8, temperature dependent mean relaxation times of 1-propanol are collected. To provide an overview over a large $T$ range, in frame (a) only data from dielectric spectroscopy are shown. In particular, we compare the time constants from the present work with those reported by Hansen et al. ${ }^{29}$ and Kudlik et al. ${ }^{49}$ We find good overall agreement for the three time scale traces identified in this plot. This applies to the relaxation times of the Debye and the $\alpha$ process, both approaching each other at high as well as at low temperatures, in accord with previous observations. ${ }^{23}$ Also for the time constants $\tau_{\beta}$, referring to the Johari-Goldstein secondary relaxation, good agreement is noted for the results from the various studies.

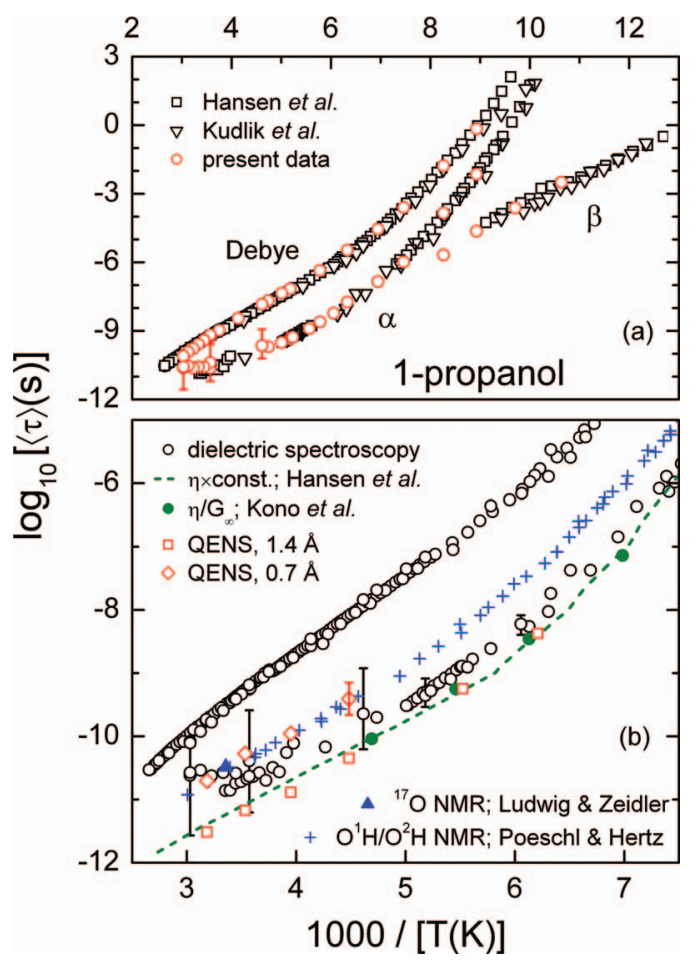

FIG. 8. (a) Arrhenius plot showing the temperature dependence of the relaxation times determined from the broad-band dielectric spectra of the present work (cf. Fig. 3) and reported in the literature (squares: Ref. 29, triangles: Ref. 49). (b) Comparison of the Debye- and $\alpha$-relaxation times obtained by different experimental methods at $\tau<10^{-5} \mathrm{~s}$. The circles show the results from dielectric spectroscopy [cf. frame (a); for better readability, in (b) the same symbols are used for literature and present data]. Some representative error bars are given for dielectric relaxation times obtained by analyzing the high-frequency flank of the loss peak (cf. Fig. 4). NMR data are shown as crosses (Ref. 78) or as a triangle (Ref. 79). The results from quasielastic neutron scattering (QENS) are plotted as open diamonds (pre-peak data) and open squares (main-peak data). The dashed line represents a fit to the shear viscosities $\eta$ collected in Ref. 29, here multiplied by a factor of $k=2.2 \times 10^{-9} \mathrm{~Pa}^{-1}$, to achieve overlap with the Maxwell relaxation times, $\tau_{\eta}=\eta / G_{\infty}$. The latter times were calculated on the basis of the highfrequency shear modulus $G_{\infty}$, taken from Ref. 27.

In the context of the present work, the high-frequency dynamics of 1-propanol is of particular interest. Therefore, in Fig. 8(b) we replotted the corresponding frequency range and added results obtained using other techniques as well. This figure includes data: (i) from the present quasi-elastic neutron scattering experiments performed at the pre-peak and at the main-peak of the structure factor, ${ }^{77}$ (ii) from NMR as measured at the hydrogen ${ }^{78}$ and the oxygen ${ }^{79}$ site of the hydroxyl group and therefore called $\tau_{\mathrm{OH}},{ }^{2}$ and (iii) from the Maxwell relationship, $\tau_{\eta}=\eta / G_{\infty}$, on the basis of the (slightly temperature dependent) high-frequency shear modulus, $G_{\infty}$, accessed via ultrasonic techniques ${ }^{27}$ and the viscosities ${ }^{80}$ collected in Ref. 29. Since $G_{\infty}$ was measured only up to $\sim 210 \mathrm{~K}$ in Ref. 27 , the viscosity multiplied by a factor, $k$, empirically chosen such that coincidence with $\tau_{\eta}$ is achieved, is also show in Fig. 8(b).

Referring to Fig. 8(b) with a focus on time scales shorter than $\tau_{D}$ we can make two important observations. On the one hand, we find that the time scales determined at the $Q$ values of the main peak are compatible with the ones relating to viscosity. This means that at the structure factor 
maximum the structural relaxation is probed, as one may have expected from similar observations made for other glass formers such as the network former glycerol ${ }^{81}$ or the van der Waals liquid ortho-terphenyl. ${ }^{82}$ We note that in monohydroxy alcohols the structural relaxation appears to stem from the motion of the molecular alkyl chains. ${ }^{30}$ On the other hand, for the three highest temperatures, at which we have pre-peak relaxation times these agree well, within experimental uncertainty, with the relaxation times $\tau_{\mathrm{OH}}$ as measured at the $\mathrm{OH}$ group by NMR techniques. ${ }^{78,79}$ These NMR results were originally analyzed with a focus on intramolecular motions, but later reinterpreted in terms of the average time an $\mathrm{OH}$ group stays in one cluster, ${ }^{2}$ which should be proportional to the average cluster size divided by the rate at which monomers join or leave it.

The dielectric relaxation times, $\tau_{\alpha}$, indicate that, on the one hand, at time scales longer than about 1 ns the structural relaxation is probed ${ }^{83}$ On the other hand, at the highest temperatures reached in the present work, the dielectric $\alpha$ relaxation times seem to approach $\tau_{\mathrm{OH}}$ if the uncertainty in their determination, discussed in relation to Fig. 4, is disregarded. However, as emphasized above, within the microwave range the components contributing to the effectively singlepeak spectral shape of the dielectric loss are hard to resolve. ${ }^{84}$ Under such circumstances a reliable determination of dielectric $\alpha$-relaxation times in the range $\tau_{\alpha}<10^{-10} \mathrm{~s}$ is rendered almost impossible as confirmed by the present results for 1propanol.

\section{B. Transient chains and cluster-cluster decorrelation}

How can the time scales characterizing the intermediate scattering functions measured at the pre-peak position be understood in terms of the molecular motion? Clearly, these time scales relate to the decay of the cluster-cluster correlation and, since translational diffusion experiments show that only single molecules move and not entire clusters, a structure diffusion mechanism ${ }^{85}$ can be invoked. ${ }^{2}$ One example of such a mechanism is embodied in the transient chain model: Here end-to-end chains of $\mathrm{OH}$ groups are subject to permanent restructuring as a consequence of a continuous attachment and detachment of molecules, preferably near the terminals of these end-to-end chains. ${ }^{2}$ By means of spin-lattice relaxation measurements the life time of a molecule within these clusters was determined and designated $\tau_{\mathrm{OH}} \cdot{ }^{2,42}$ As Fig. 8 shows, this time scale coincides with the time scale measured at the pre-peak.

It has long been suggested ${ }^{20}$ and was recently reemphasized $^{86,87}$ that monohydroxy alcohols might be classified into two groups: Group I, to which 1-propanol belongs, was defined to include liquids with strong (Debye-type) dielectric relaxation processes for which chain-like molecular association dominates. Liquids of group II, on the other hand, display rather low dielectric strengths, suggesting that ring-like associates with close to vanishing effective electrical dipole moments are prevalent. It would be interesting to perform quasi-elastic neutron scattering experiments also for group II alcohols because a behavior rather different from that found for 1-propanol may be expected. As for worm-like micelles, ${ }^{88}$ ring-like supramolecular structures do not "automatically" move (i.e., translationally diffuse) when a molecule leaves or enters such a structure which implies that the cluster-cluster decay time should be relatively long. On the other hand, in ring forming systems such as 4-methyl3-heptanol the time scale separation between Debye-like and structural relaxation is much smaller than for 1-propanol. ${ }^{23}$ To minimize systematic errors under such circumstances it may turn out necessary to perform simultaneous dielectric and neutron experiments as, e.g., was done in crystallization studies of monohydroxy alcohols. ${ }^{89}$

\section{Hydrogen bond cooperativity}

The strengthening of $\mathrm{H}$ bonds in the presence of additional $\mathrm{H}$ bonds in close spatial proximity with each other is called hydrogen bond cooperativity. ${ }^{90}$ In simple terms, the increase of the mutual charge separation induced by this proximity increases the electrostatic interaction within the cluster, which can lead to stabilized hydrogen bonds. The effect typically depends on the number of bonds that effectively interact with each other. For several monohydroxy alcohols the importance of cooperative effects has been emphasized in relation to the observations that the absorbance ratio and the dielectric constant display significant changes in their temperature dependence, ${ }^{23,64}$ see also Fig. 7(a). This feature was recently interpreted in terms of a progressive destabilization of end-to-end chains as temperature is increased. ${ }^{30}$ This kind of destabilization does not seem to be complete even at room temperature, because on the basis of the Kirkwood factor, ${ }^{91}$ a measure of the mutual alignment of molecular dipoles and assessed from the total dielectric strength, significant molecular associations have been reported to persist even for superheated alcohols. ${ }^{92}$

The change of slope observed in the temperature dependence of the absorbance ratio of 1-propanol, see Fig. 7(a), and several other monohydroxy alcohols ${ }^{23,93}$ can be understood as a change in the enthalpy, $\Delta H$, see Table I. Below about 200 to $250 \mathrm{~K}$ enthalpies $\Delta H$ of $1-1.5 \mathrm{~kJ} / \mathrm{mol}$ are found for several monohydroxy alcohols. ${ }^{23}$ In analogy to Ref. 23 we interpret the relatively small $\Delta H$ values for 1-propanol as indicative of the existence of an equilibrium between enthalpically similar supramolecular structures. Above about 200 to $250 \mathrm{~K}$ the effective enthalpies are strongly temperature dependent. In the range specified in Table I an enthalpy of about $20 \mathrm{~kJ} / \mathrm{mol}$ is found for propanol. In Ref. 23 which focused on temperatures $<300 \mathrm{~K}$ smaller apparent enthalpy values were reported which may well be compatible with those for propanol after taking into account more high-temperature NIR data.

Interestingly, with $n$ denoting the number of $\mathrm{OH}$ groups per molecule, for the polyhydric alcohols listed in Table I we find that $\Delta H / n$ is roughly constant and about equal to $\Delta H$ for the monohydroxy alcohols in their low-temperature regime. The question of why in spite of the supposedly rather different cluster or network reorganizations of the monohydroxy vs. polyhydric alcohols these values are so similar deserves further study. One line of thought to rationalize this surprising finding may be found in Ref. 1: There it was observed that while the molecular networks are very different when 
going from monohydroxy to poly-alcohols, the individual $\mathrm{OH}$-networks (i.e., what one gets if one removes everything but the $\mathrm{O}$ and the $\mathrm{H}$ atoms) are very similar to the ones in monohydroxy alcohols.

\section{SUMMARY}

We have studied: static and dynamic properties of 1propanol (i) using quasi-elastic neutron scattering including momentum transfers $Q$ in the main and pre-peak regions of the structure factor, (ii) near-infrared spectroscopy focusing on the overtones of the $\mathrm{OH}$ stretching vibrations, and (iii) broad-band dielectric spectroscopy carried out up to $40 \mathrm{GHz}$. For a range of temperatures the relaxation times assessed from the intermediate scattering functions at the main peak coincide with those estimated on the basis of the Maxwell relation from viscosimetric data. High-frequency dielectric measurements fail to determine these latter relaxation times reliably, which was demonstrated on the basis of a careful analysis of the corresponding relatively unstructured high-temperature dielectric spectra. Our new permittivity data were measured in an extended frequency range with state-of-the-art precision in order to obtain realistic error estimates. The time scale determined in the pre-peak region coincides with findings from previous NMR experiments. ${ }^{2}$ Furthermore, we demonstrate that a change in the temperature dependent absorbance ratio and dielectric constant, previously reported for several monohydroxy alcohols, is found for 1-propanol as well. Importantly, we show that this "anomaly" is not observed for the corresponding polyalcohols, as determined from temperature dependent NIR spectra on propylene glycol and glycerol.

\section{ACKNOWLEDGMENTS}

Support of this project by the Deutsche Forschungsgemeinschaft under Grant No. BO1301/8-2, via Research Unit FOR1394, and from the Swedish Research Council is gratefully acknowledged.

${ }^{1}$ P. Sillrén, J. Bielecki, J. Mattsson, L. Börjesson, and A. Matic, J. Chem. Phys. 136, 094514 (2012).

${ }^{2}$ C. Gainaru, R. Meier, S. Schildmann, C. Lederle, W. Hiller, E. Rössler, and R. Böhmer, Phys. Rev. Lett. 105, 258303 (2010).

${ }^{3}$ M. Paolantoni, P. Sassi, A. Morresi, and R. Cataliotti, Chem. Phys. 310, 169 (2005); F. Palombo, P. Sassi, M. Paolantoni, and A. Morresi, J. Phys. Chem. B 110, 18017 (2006); M. Paolantoni, P. Sassi, A. Morresi, and R. Cataliotti, J. Raman Spectrosc. 37, 528 (2006).

${ }^{4}$ V. V. Levin and Y. D. Feldman, Chem. Phys. Lett. 87, 162 (1982).

${ }^{5}$ A. Soper, Chem. Phys. 202, 295 (1996).

${ }^{6}$ I. Akiyama, M. Ogawa, K. Takase, T. Takamuku, T. Yamaguchi, and N. Ohtori, J. Solution Chem. 33, 797 (2004).

${ }^{7}$ A. Mikusinska-Planner, Acta Crystallogr., Sect. A 33, 433 (1977).

${ }^{8}$ E. Tsuchida, Y. Kanada, and M. Tsukada, Chem. Phys. Lett. 311, 236 (1999).

${ }^{9}$ H. Torii, Pure Appl. Chem. 76, 247 (2004).

${ }^{10}$ T. Kosztolanyi, I. Bakó, and G. Pálinkás, J. Chem. Phys. 118, 4546 (2003).

${ }^{11}$ K. Vahvaselka, R. Serimaa, and M. Torkkeli, J. Appl. Crystallogr. 28, 189 (1995).

${ }^{12}$ H. Graener, T. Q. Ye, and A. Laubereau, J. Chem. Phys. 90, 3413 (1989); 91, 1043 (1989).

${ }^{13}$ L. Saiz, J. A. Padró, and E. Guàrdia, J. Phys. Chem. B 101, 78 (1997); J. A. Padró, L. Saiz, and E. Guàrdia, J. Mol. Struct. 416, 243 (1997).

${ }^{14}$ M. Haughney, M. Ferrario, and I. McDonald, J. Phys. Chem. 91, 4934 (1987).
${ }^{15}$ I. Svishchev and P. Kusalik, J. Chem. Phys. 100, 5165 (1994).

${ }^{16}$ S. W. Benson, J. Am. Chem. Soc. 118, 10645 (1996).

${ }^{17}$ A. Sahoo, S. Sarkar, V. Bhagat, and R. N. Joarder, J. Phys. Chem. A 113, 5160 (2009).

${ }^{18}$ R. Ludwig, Chem. Phys. Chem. 6, 1369 (2005).

${ }^{19}$ P. Sillrén, J. Swenson, J. Mattsson, D. Bowron, and A. Matic, J. Chem. Phys. 138, 214501 (2013).

${ }^{20}$ W. Dannhauser, J. Chem. Phys. 48, 1911 (1968).

${ }^{21}$ S. S. N. Murthy and M. Tyagi, J. Chem. Phys. 117, 3837 (2002).

${ }^{22}$ L. P. Singh, C. Alba-Simionesco, and R. Richert, J. Chem. Phys. 139, 144503 (2013).

${ }^{23}$ S. Bauer, K. Burlafinger, C. Gainaru, S. Kastner, F. Mayr, P. Lunkenheimer, W. Hiller, A. Loidl, and R. Böhmer, J. Chem. Phys. 138, 094505 (2013).

${ }^{24} \mathrm{~W}$. Dannhauser and R. Cole, J. Chem. Phys. 23, 1762 (1955).

${ }^{25}$ M. A. Floriano and C. A. Angell, J. Chem. Phys. 91, 2537 (1989).

${ }^{26}$ J. Barthel, K. Bachhuber, R. Buchner, and H. Hetzenauer, Chem. Phys. Lett. 165, 369 (1990).

${ }^{27}$ R. Kono, T. A. Litovitz, and G. E. McDuffie, J. Chem. Phys. 45, 1790 (1966).

${ }^{28}$ H. Huth, L.-M. Wang, C. Schick, and R. Richert, J. Chem. Phys. 126, 104503 (2007).

${ }^{29}$ C. Hansen, F. Stickel, T. Berger, R. Richert, and E. W. Fischer, J. Chem. Phys. 107, 1086 (1997).

${ }^{30}$ C. Lederle, W. Hiller, C. Gainaru, and R. Böhmer, J. Chem. Phys. 134, 064512 (2011)

${ }^{31}$ Y. Gao, D. Bi, X. Li, R. Liu, Y. Tian, and L.-M. Wang, J. Chem. Phys. 139, 024503 (2013).

${ }^{32}$ T. El Goresy and R. Böhmer, J. Chem. Phys. 128, 154520 (2008).

${ }^{33}$ B. Jakobsen, C. Maggi, T. Christensen, and J. C. Dyre, J. Chem. Phys. 129, 184502 (2008).

${ }^{34} \mathrm{P}$. Debye, "Zur Theorie der anomalen Dispersion im Gebiete der langwelligen elektrischen Strahlung," Verh. Dtsch. Phys. Ges. 15, 777 (1913); Polar Molecules (Chemical Catalog Company, New York, 1929).

${ }^{35}$ H. Weingärtner, H. Nadolny, A. Oleinikova, and R. Ludwig, J. Chem. Phys. 120, 11692 (2004).

${ }^{36}$ F. J. Bermejo, W. S. Howells, M. Jimenez-Ruiz, M. A. Gonzalez, D. L. Price, M. L. Saboungi, and C. Cabrillo, Phys. Rev. B 69, 174201 (2004).

${ }^{37}$ Y. Takagi, T. Yano, M. Mikami, and S. Kojima, Physica B 263-264, 306 (1999).

${ }^{38}$ J.-H. Ko and S. Kojima, Jpn. J. Appl. Phys. 41, 3206 (2002).

${ }^{39}$ T. Lyon and T. A. Litovitz, J. Appl. Phys. 27, 179 (1956); T. A. Litovitz and G. E. McDuffie, Jr., J. Chem. Phys. 39, 729 (1963).

${ }^{40}$ R. Behrends and U. Kaatze, J. Phys. Chem. A 105, 5829 (2001).

${ }^{41}$ C. Gainaru, R. Figuli, T. Hecksher, B. Jakobsen, J. C. Dyre, M. Wilhelm, and R. Böhmer, Phys. Rev. Lett. 112, 098301 (2014).

${ }^{42}$ S. Schildmann, A. Reiser, R. Gainaru, C. Gainaru, and R. Böhmer, J. Chem. Phys. 135, 174511 (2011).

${ }^{43}$ G. W. Stewart and R. M. Morrow, Phys. Rev. 30, 232 (1927).

${ }^{44}$ A. Vrhovšek, O. Gereben, S. Pothoczki, M. Tomšič, A. Jamnik, S. Kohara, and L. Pusztai, J. Phys.: Condens. Matter 22, 404214 (2010).

${ }^{45}$ P. Zetterström, U. Dahlborg, and W. S. Howells, Mol. Phys. 81, 1187 (1994); P. Zetterström, U. Dahlborg, and A. Wannberg, ibid. 83, 971 (1994).

${ }^{46}$ A. Faraone, K. Hong, L. R. Kneller, M. Ohl, and J. R. D. Copley, J. Chem. Phys. 136, 104502 (2012).

${ }^{47}$ R. Böhmer, C. Gainaru, and R. Richert, "Structure and dynamics of monohydroxy alcohols - Milestones towards their microscopic understanding, 100 years after Debye," Phys. Rep. (unpublished).

${ }^{48}$ B. Schiener and R. Böhmer, J. Non-Cryst. Solids 182, 180 (1995).

${ }^{49}$ A. Kudlik, C. Tschirwitz, S. Benkhof, T. Blochowicz, and E. Rössler, Europhys. Lett. 40, 649 (1997).

${ }^{50}$ G. Power, G. P. Johari, and J. K. Vij, J. Chem. Phys. 116, 4192 (2002).

${ }^{51}$ Y. Hiejima and M. Yao, J. Chem. Phys. 119, 7931 (2003).

${ }^{52}$ K. Z. Haufa and M. A. Czarnecki, Vib. Spectrosc. 51, 80 (2009).

${ }^{53}$ K. Izutsu, Y. Hiyama, C. Yomota, and T. Kawanischi, AAPS PharmSciTech 10, 524 (2009).

${ }^{54}$ R. Böhmer, M. Maglione, P. Lunkenheimer, and A. Loidl, J. Appl. Phys. 65, 901 (1989).

${ }^{55}$ Y. Z. Wei and S. Sridhar, Rev. Sci. Instrum. 60, 3041 (1989).

${ }^{56}$ G. Q. Jiang, W. H. Wong, E. Y. Raskovich, W. G. Clark, W. A. Hines, and J. Sanny, Rev. Sci. Instrum. 64, 1614 (1993).

${ }^{57}$ Apart from the neutron data on deuterated 1-propanol, for which a polarization analysis confirms that in the pre-peak region mainly coherent scattering occurs, we also investigated protonated 1-propanol, giving a purely 
incoherent signal. We find a large difference between the dynamics seen in the incoherent and in the coherent scattering. For protonated 1-propanol the measured time scales follow a simple $Q^{-2}$ law. The self-diffusivity calculated from fits to these data agrees pretty well with the NMR self-diffusion coefficients reported by S. Meckl and M. D. Zeidler, Mol. Phys. 63, 85 (1988). For deuterated 1-propanol the $Q$ dependence of the time scales is much stronger at temperatures at which it can reliably be determined, see also the discussion in Sec. IV A.

${ }^{58}$ G. P. Johari and M. Goldstein, J. Chem. Phys. 53, 2372 (1970).

${ }^{59}$ A. Kudlik, S. Benkhof, T. Blochowicz, C. Tschirwitz, and E. A. Rössler, J. Mol. Struct. 479, 201 (1999).

${ }^{60}$ K. L. Ngai and M. Paluch, J. Chem. Phys. 120, 857 (2004).

${ }^{61}$ S. Kastner, M. Köhler, Y. Goncharov, P. Lunkenheimer, and A. Loidl, J. Non-Cryst. Solids 357, 510 (2011).

${ }^{62} \mathrm{P}$. Lunkenheimer, M. Köhler, S. Kastner, and A. Loidl, in Structural Glasses and Supercooled Liquids: Theory, Experiment, and Applications, edited by P. G. Wolynes and V. Lubchenko (Wiley, Hoboken, 2012), Chap. 3, p. 115.

${ }^{63}$ C. J. F. Böttcher and P. Bordewijk, Theory of Electric Polarization (Elsevier, Amsterdam, 1973), Vol. II.

${ }^{64}$ C. Gainaru, S. Kastner, F. Mayr, P. Lunkenheimer, S. Schildmann, H. J. Weber, W. Hiller, A. Loidl, and R. Böhmer, Phys. Rev. Lett. 107, 118304 (2011).

${ }^{65}$ Broadband Dielectric Spectroscopy, edited by F. Kremer and A. Schönhals (Springer, Heidelberg, 2003).

${ }^{66}$ Moreover, one should note that the justification for using a simple additive superposition of different contributions to $\varepsilon^{*}(v)$ may be doubted, see, e.g., R. Böhmer, G. Diezemann, B. Geil, G. Hinze, A. Nowaczyk, and M. Winterlich, Phys. Rev. Lett. 97, 135701 (2006). Alternatives were promoted in A. Arbe, D. Richter, J. Colmenero, and B. Farago, Phys. Rev. E 54, 3853 (1996); see also E. Donth, K. Schröter, and S. Kahle, Phys. Rev. E 60, 1099 (1999). However, usually the convolution approach leads to similar results as the additive approach, see, e.g., P. Lunkenheimer et al., Phys. Rev. E 77, 031506 (2008), especially if the relaxation times of the analyzed processes differ significantly, which indeed is the case for 1-propanol at least for temperatures $<330 \mathrm{~K}$ (cf. Fig. 8).

${ }^{67}$ Infrared data were previously published in a more restricted temperature range by, e.g., N. Michniewicz, M. A. Czarnecki, and J. P. Hawranek, J. Mol. Struct. 844-845, 181 (2007).

${ }^{68} \mathrm{~J}$. Workman and L. Weyer, Practical Guide to Interpretive Near-Infrared Spectroscopy (CRC, Boca Raton, 2008), p. 29.

${ }^{69} \mathrm{~A}$ more detailed assignment of these bands may be possible on the basis of suitable quantum chemical calculations, see, e.g., K. Ohno, T. Shimoaka, N. Akai, and Y. Katsumoto, J. Phys. Chem. A 112, 7342 (2008).

${ }^{70}$ S. Takahara, O. Yamamuro, and H. Suga, J. Non-Cryst. Solids 171, 259 (1994).

${ }^{71}$ A. Barkatt and C. A. Angell, J. Chem. Phys. 70, 901 (1979).
${ }^{72}$ M. Köhler, P. Lunkenheimer, Y. Goncharov, R. Wehn, and A. Loidl, J. Non-Cryst. Solids 356, 529 (2010).

${ }^{73}$ P. Papadopoulos, W. Kossack, and F. Kremer, Soft Matter 9, 1600 (2013).

${ }^{74}$ E. Donth, J. Korus, E. Hempel, and M. Beiner, Thermochim. Acta 304305, 239 (1997).

${ }^{75}$ P. Lunkenheimer and A. Loidl, Chem. Phys. 284, 205 (2002).

${ }^{76}$ J. D. Worley and I. M. Klotz, J. Chem. Phys. 45, 2868 (1966).

${ }^{77} \mathrm{~A}$ previous quasi-elastic neutron scattering study reported correlation times for propanol, see Ref. 36. The analysis relied on a number of assumptions and yielded time scales (see their Fig. 9) which display a completely different temperature dependence than all other time scales shown in Fig. 8 of the present article. The results from Ref. 36 are not included in our Arrhenius plot.

${ }^{78}$ M. Pöschl and H. G. Hertz, J. Phys. Chem. 98, 8195 (1994).

${ }^{79}$ R. Ludwig and M. D. Zeidler, Mol. Phys. 82, 313 (1994).

${ }^{80}$ The compilation in Ref. 29 is based on the following articles: G. Tammann and W. Hesse, Z. Anorg. Allg. Chem. 156, 245 (1926); E. N. C. Andrade, Philos. Mag. 17, 698 (1934); D. J. Denney, J. Chem. Phys. 30, 159 (1959); A. C. Ling and J. E. Willard, J. Phys. Chem. 72, 1918 (1968).

${ }^{81}$ K. E. Larsson, Phys. Rev. 167, 171 (1968). See the compilation in U. Schneider, Breitbandige dielektrische Studien der Dynamik struktureller Glasbildner (Books on Demand, Norderstedt, 2000).

${ }^{82}$ See Fig. 26 in A. Tölle, Rep. Prog. Phys. 64, 1473 (2001).

${ }^{83}$ In Fig. 8(b) the mechanical time constants appear to be slightly smaller than the dielectric ones. Such a deviation of modulus vs. susceptibility based time constants is not uncommon, see, e.g., B. Jakobsen, T. Hecksher, T. Christensen, N. B. Olsen, J. C. Dyre, and K. Niss, J. Chem. Phys. 136, 081102 (2012).

${ }^{84} \mathrm{This}$ was noted for $2 \mathrm{E} 1 \mathrm{H}$ at $T>310 \mathrm{~K}$ in Ref. 42 with reference to the data in Ref. 2.

${ }^{85}$ D. Gerritzen and H. H. Limbach, Ber. Bunsen-Ges. Phys. Chem. 85, 527 (1981).

${ }^{86}$ G. P. Johari, J. Chem. Phys. 139, 026101 (2013).

${ }^{87}$ S. Pawlus, M. Wikarek, C. Gainaru, M. Paluch, and R. Böhmer, J. Chem. Phys. 139, 064501 (2013); S. Bauer, H. Wittkamp, S. Schildmann, M. Frey, W. Hiller, T. Hecksher, N. B. Olsen, C. Gainaru, and R. Böhmer, ibid. 139, 134503 (2013).

${ }^{88}$ See, e.g., R. Granek and M. E. Cates, J. Chem. Phys. 96, 4758 (1992).

${ }^{89}$ See, e.g., A. Sanz, A. Nogales, I. Puente-Orench, M. Jimenez-Ruiz, and T. A. Ezquerra, Phys. Rev. Lett. 107, 025502 (2011).

${ }^{90}$ A. Karpfen, "Cooperative effects in hydrogen bonding," Adv. Chem. Phys. 123, 469 (2002).

${ }^{91}$ C. J. F. Böttcher, Theory of Electric Polarization (Elsevier, Amsterdam, 1973), Vol. I.

${ }^{92}$ W. Dannhauser and L. W. Bahe, J. Chem. Phys. 40, 3058 (1964).

${ }^{93}$ A. Hédoux, Y. Guinet, L. Paccou, P. Derollez, and F. Danède, J. Chem. Phys. 138, 214506 (2013), see their Fig. 7. 